\title{
Tailoring the spectral response of add/drop single and multiple resonators in silicon-on-insulator
}

\author{
Invited Paper \\ B. Timotijevic ${ }^{1}$, G. Mashanovich ${ }^{1}$, A. Michaeli ${ }^{2}$, O. Cohen ${ }^{2}$, V. M. N. Passaro ${ }^{3}$, \\ J. Crnjanski ${ }^{4}$, and G. T. Reed ${ }^{1 *}$ \\ ${ }^{1}$ Advanced Technology Institute, University of Surrey, Guildford, GU2 7XH, UK \\ (B. Timotijevic is now with CSEM SA, Rue Jaquet-Droz 1, CH-2002 Neuchatel, Switzerland) \\ ${ }^{2}$ Intel Corporation, Kyriat Gat 82109, Jerusalem, Israel \\ ${ }^{3}$ Politecnico di Bari, via E. Orabona 4, 70125 Bari, Italy \\ ${ }^{4}$ Faculty of Electrical Engineering, University of Belgrade, Bulevar kralja \\ Aleksandra 73b, 11120 Belgrade, Serbia \\ ${ }^{*}$ E-mail: g.reed@surrey.ac.uk \\ Received February 13, 2009
}

\begin{abstract}
Channel dropping waveguide filters based on single and multiple resonators in silicon-on-insulator (SOI) technology are of great interest due to their compactness and high wavelength selectivity, which is a desirable feature for photonic modulators, detectors, and other optically integrated components in telecommunication systems, in particular for wavelength division multiplexing (WDM) systems. Particular advantage of these filters is that they are capable of producing relatively large free spectral range (FSR) as well as narrow 3-dB bandwidth of the filter resonances. Herein we report experimental results and discuss the possibility of designing mono-mode and (nearly) polarization independent SOI ring and racetrack resonators with the FSR in excess of $30 \mathrm{~nm}$.
\end{abstract}

OCIS codes: $130.0130,250.0250,230.7370,260.3060,260.5430$

doi: $10.3788 / C O L 20090704.0291$.

Miniaturization of photonic devices has been significantly intensified in the last decade in an attempt to improve a footprint and performance of integrated optical components. Silicon-on-insulator (SOI) has emerged as a promising material choice for various integrated optoelectronic devices ${ }^{[1]}$. It is attractive for complex optical systems as the cost can be significantly reduced due to the compatibility with complementary metal oxide semiconductor (CMOS) technology ${ }^{[2]}$. It also has a high refractive index contrast between the core and the cladding, which is an important property for good confinement of light and efficient guiding and coupling in sub-micron waveguides. However, for those devices that are intended to be part of broadband optical networks, for example multiplexers and de-multiplexers, it is desirable to demonstrate a high selectivity and a tunable response. Thus, it is necessary to provide wavelength selective elements with the ability to filter input data streams producing a large free spectral range (FSR), a small full-width at half-maximum (FWHM), and a high quality factor $(Q)$, and all conditions set by communication standards. Owing to the generic and adaptable operation, ring-resonator-types of filters in SOI are often considered as candidates to meet these demands.

In silicon photonics, there is a particular focus on two waveguide architectures upon which the devices are typically built. Rib waveguides have proven to be particularly useful regarding polarization properties ${ }^{[3]}$. Single-mode and polarization independent (PI) resonators on rib waveguides have already been experimentally demonstrated ${ }^{[4]}$, but their FSR is usually small for applications in optical networks. On the other hand, strip waveguides, or photonic wires, allow small bend radii ${ }^{[5,6]}$, which in turn results in the improved FSR. They have to be rather small in cross-section to prevent higher-order modes (HOMs) from propagation ${ }^{[7]}$, and they exhibit polarization dependence and loss issues.

In majority of applications, single-mode devices are required in order to minimize dispersion caused by multiple spatial modes which, in turn, allows for more information to be transmitted per unit time giving a higher bandwidth. It is also desirable that TE and TM modes result in similar transfer functions, i.e., a filter should perform as a PI device as, otherwise, two separate devices, each optimized for operation for one particular polarization, need to be provided ${ }^{[8]}$.

In this letter, the structures are composed of Siwaveguide and $\mathrm{SiO}_{2}$ top and bottom claddings. Furthermore, the waveguide height (WGH) is a fixed parameter, dictated in advance by established wafer processing procedures. The rib WGH is set to $1.35 \mu \mathrm{m}$ and the strip waveguides WGH $=0.29$ or $0.34 \mu \mathrm{m}$, while the waveguide width (WGW) and the etch depth (ED) are variables used to target the best performance.

Single-mode rib waveguides with relatively large cross sections have been studied extensively by a number of researchers to find single-mode behavior at the same time as low propagation $\operatorname{loss}^{[9,10]}$. They were recognized as promising candidates due to the compatibility with single-mode fiber dimensions and the possibility to control the polarization. The single-mode behavior and PI propagation depend both on geometrical parameters of the waveguide (WGH, WGW, ED, sidewall angle) 
and on stress caused by the cladding (typically silicon dioxide) ${ }^{[11]}$.

In Ref. [4], racetrack resonators built upon rib waveguides instead of rings are used to demonstrate PI response as they allow for equal propagation of TE and TM modes in a straight section, also known as a directional coupler. More precisely, TE mode propagates faster in this area and the length of the directional coupler is adjusted to allow for multiple (odd) transitions of TM polarization and to achieve PI in this way. The alignment of $2 \mathrm{pm}$ and $Q$ factor of 90000 were achieved over a FSR of $0.19 \mathrm{~nm}^{[4]}$. The racetrack resonators have an additional advantage over the ring resonators in improved coupling efficiency as the curvature of the ring is quite short. However, the racetracks occupy larger area than the rings and due to the straight section, the FSR of a racetrack resonator is limited. To improve the coupling efficiency of a ring without adding length to the resonator, a bend waveguide for coupling can be used ${ }^{[12]}$.

Better alignment of TE and TM responses over larger FSR is achieved in this letter by using lattice coupled racetrack resonators. The cross-sectional geometry of the rib waveguides is similar to those used in Ref. [4], but a smaller circumference of resonators is employed, together with multi-stage configuration in order to enhance FSR and polarization response respectively. Figure 1(a) demonstrates a PI response, with the alignment within $3 \mathrm{pm}$ at the FSR of $0.5 \mathrm{~nm}$ around the wavelength of $1.55 \mu \mathrm{m}$, for a filter that consists of two similar rib racetracks with $\mathrm{WGW}=0.8 \mu \mathrm{m}$, ring radii of $r_{1}=100 \mu \mathrm{m}$ and $r_{2}=99.5 \mu \mathrm{m}$, and the length of the straight section of $l=310 \mu \mathrm{m}$. To demonstrate the polarization independence more clearly, both graphs are shown in absolute values. The FSR is more than twice larger than the one reported in Ref. [4]. The responses were not reproduced at the drop port as shown in Fig. 1(b), probably because of the modified propagating conditions for TE and TM modes in the upper resonator. This could not be noticed

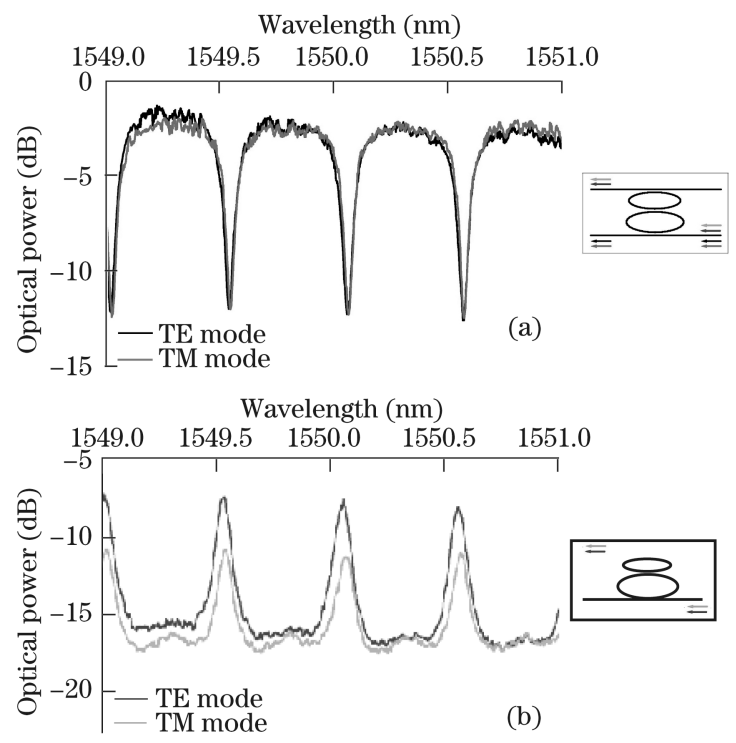

Fig. 1. TE and TM spectral responses at the (a) through and (b) drop ports of a lattice filter consisting of two similar rib racetracks. WGW $=0.8 \mu \mathrm{m}, r_{1}=100 \mu \mathrm{m}, r_{2}=99.5 \mu \mathrm{m}$, and $l=310 \mu \mathrm{m}$.

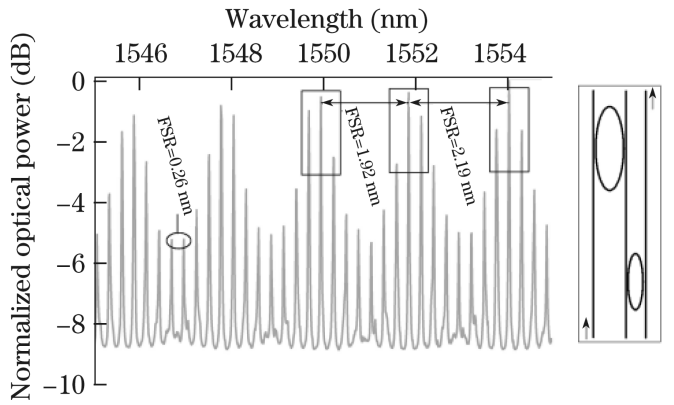

Fig. 2. TM spectral response at the drop port of a cascaded filter consisting of dissimilar racetracks. WGW $=1.0 \mu \mathrm{m}, l$ $=1030 \mu \mathrm{m}, r_{1}=100 \mu \mathrm{m}$, and $r_{2}=50 \mu \mathrm{m}$.

at the through port response due to the relatively small interstitial coupling coefficient, i.e., due to the negligible influence of the upper on the bottom resonator.

These structures were also used to investigate the possibility of improving the FSR through the Vernier effect ${ }^{[13]}$. Two single resonators with the corresponding FSRs of $\mathrm{FSR}_{1}$ and $\mathrm{FSR}_{2}$ when coupled, i.e., placed closely to each other, give several times better FSR, that is: FSR $=m \times$ $\mathrm{FSR}_{1}=n \times \mathrm{FSR}_{2}$, where $m$ and $n$ are integers. It can be speculated that a very large overall FSR can be achieved provided that $\mathrm{FSR}_{1}$ and $\mathrm{FSR}_{2}$ have different but still very similar values of circumferences. The experiments have shown that the Vernier effect is difficult to satisfy in that case and that the whole structure is more likely to act as a single device, i.e., to give single-resonator type of response as shown in Fig. 1. From series of experiments, it was concluded that resonators need to be much more dissimilar to make this effect visible, as shown in Fig. 2. In this example, two racetrack resonators with radii of 100 and $50 \mu \mathrm{m}$ and coupler length of $1.03 \mathrm{~mm}$ were used in cascaded configuration. It can be seen that single resonator FSR of $0.29 \mathrm{~nm}$ can be potentially improved to approximately $2 \mathrm{~nm}$, but the side-lobes still need to be addressed through finer adjustments of device geometry.

Possible issues in these devices are polarization conversion $^{[14]}$ and existence of higher-order modes. Figure 3 shows the measured TE spectral response at the drop port of a double-racetrack resonator when it is excited with the input $\mathrm{TE}$ polarization (TE response), and TE response of the same device when it is excited with $\mathrm{TM}$ polarization ( $\mathrm{TM} \rightarrow \mathrm{TE}$ response) in order to estimate the polarization conversion. It can be seen that

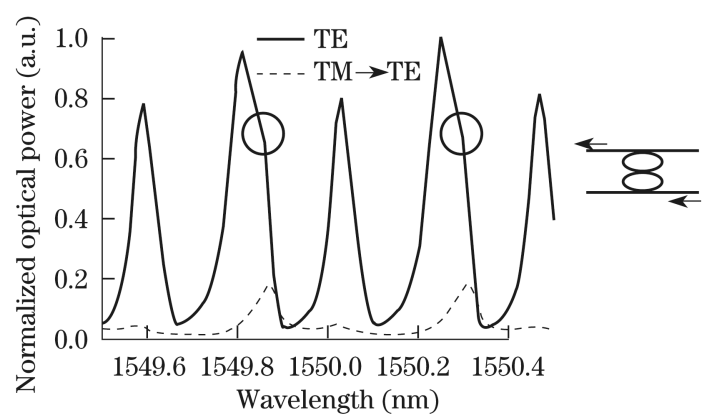

Fig. 3. Demonstration of the polarization conversion on double-racetrack resonator consisting of racetracks with $\mathrm{WGW}=1.0 \mu \mathrm{m}, l=500 \mu \mathrm{m}$, separation $=0.4 \mu \mathrm{m}$, and $r=$ $75 \mu \mathrm{m} . \mathrm{TM} \rightarrow \mathrm{TE}$ denotes the measured TE spectral response at the output for the input TM excitation. 
the converted signal $(\mathrm{TM} \rightarrow \mathrm{TE})$, the strength of which is about $20 \%$ of the TE mode, has visibly broadened the TE spectral response.

An example where extra peaks appear due to multimode behavior is shown in Fig. 4, together with some modal splitting. The two graphs represent the measured through and drop spectral responses of the TE and $\mathrm{TM} \rightarrow \mathrm{TE}$ polarizations (TE response measured at the output when TM polarization is applied at the input). The device is a double-racetrack resonator waveguide with the width of $1.0 \mu \mathrm{m}$, length of the directional coupler of $1030 \mu \mathrm{m}$, separation between resonator and the coupler of $0.58 \mu \mathrm{m}$, and radii of racetrack curvatures of $100 \mu \mathrm{m}$. Figure 4(a) shows that the polarization conversion is negligible. For this set of devices, the polarization conversion was measured to be below $5 \%$. A double filter in single-mode waveguides should give no more than two resonances in a spectral response, while we can observe three peaks (dips) in the spectral responses of the device. Therefore, one of the peaks is probably the result of the first HOM propagation. The TE spectral response at the drop port is depicted in Fig. 4(b) at the corresponding through port. The profiles of both graphs and the modelling performed prior to the fabrication suggest that the two closely spaced resonances should be the fundamental mode resonances of the double resonator, and the third resonance is a result of the first HOM propagation.

The structures discussed previously have a circumference of $1 \mathrm{~mm}$ or more, which does not allow for the FSR above $1 \mathrm{~nm}$. This is due to the large bend loss of rib waveguides if the bend radius is smaller than about 10 $\mu \mathrm{m}$. A smaller structure such as a $2-\mu \mathrm{m}$ ring resonator would provide further enhancement of the FSR, to values larger than $30 \mathrm{~nm}$, but it requires a different topology of a waveguide, the so-called silicon strip or photonic wire waveguide.

It has been shown that small waveguides with small bending radii can improve the characteristics of photonic devices, for example, optical modulators and filters $^{[15-17]}$. Furthermore, such small waveguides or
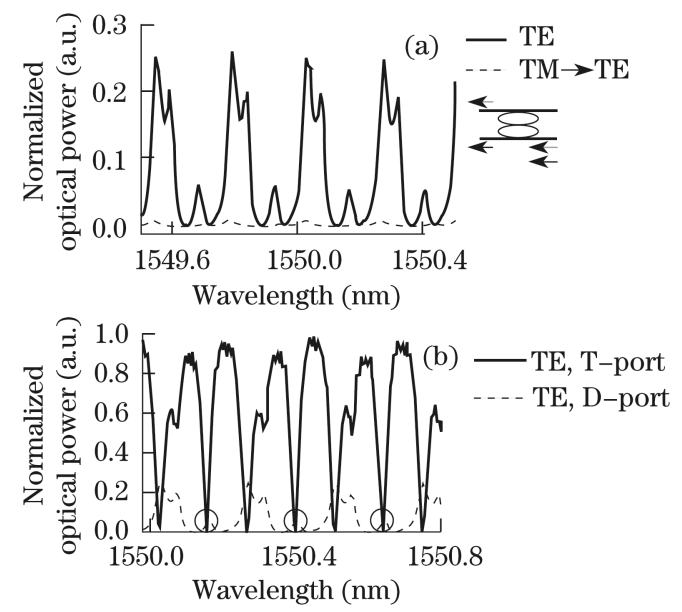

Fig. 4. Demonstration of a multimode response on doubleracetrack resonator made of racetracks with $\mathrm{WGW}=1.0 \mu \mathrm{m}$, $l=1030 \mu \mathrm{m}$, separation $=0.58 \mu \mathrm{m}$, and $r=100 \mu \mathrm{m}$. (a) $\mathrm{TE}$ and $\mathrm{TM} \rightarrow \mathrm{TE}$ spectral responses at the drop port, (b) TE response from (a) together with the corresponding response at the through port. photonic wires as they are usually called, can realize an ultra-high optical power density, which can be as much as 1000 times of that in a conventional single-mode fiber, enhancing nonlinear optical effects ${ }^{[18]}$. Unlike rib waveguides which can be monomodal even for cross sectional dimensions of several microns, dimensions of a strip waveguide must be significantly smaller than $1 \mu \mathrm{m}$ to suppress the propagation of HOMs. For example, by simulating the fundamental mode and the first two HOMs for the waveguide with a height of $220 \mathrm{~nm}$ and top oxide cover of $200 \mathrm{~nm}$, the WGW that corresponds to the single/multimode boundary was found to be $500 \mathrm{~nm}$. The propagation loss of TE modes is typically of the order of $3 \mathrm{~dB} / \mathrm{cm}$ at the wavelength of $1550 \mathrm{~nm}$ (e.g., $3.6 \mathrm{~dB} / \mathrm{cm}$ for $220 \times 445(\mathrm{~nm})$ waveguides in Ref. [5], $2.8 \mathrm{~dB} / \mathrm{cm}$ for $200 \times 400(\mathrm{~nm})$ waveguides in Ref. [19], and 2.4 $\mathrm{dB} / \mathrm{cm}$ for $220 \times 500(\mathrm{~nm})$ waveguides in Ref. [20]). Strip waveguides used for the ring and racetrack resonators in this letter had typical propagation losses for TE polarization of $3 \mathrm{~dB} / \mathrm{cm}$ for $\mathrm{WGW}=0.34 \mu \mathrm{m}$ and $4 \mathrm{~dB} / \mathrm{cm}$ for $\mathrm{WGW}=0.30 \mu \mathrm{m}^{[21]}$. These figures could be decreased further by sacrificial oxidation of the sidewalls, which smooths the sidewalls ${ }^{[22]}$. Lee et al. used both oxidation smoothing and anisotropic etching and measured a propagation loss of only $0.8 \mathrm{~dB} / \mathrm{cm}$ for single-mode strip waveguides that had a width of $500 \mathrm{~nm}^{[23]}$. Vlasov et al. also measured losses per $90^{\circ}$ bend for $220 \times 445(\mathrm{~nm})$ strip waveguides and obtained $0.005,0.013$, and 0.086 $\mathrm{dB} /$ turn for bending radius of 5,2 , and $1 \mu \mathrm{m}$, respectively ${ }^{[5]}$. Bogaerts et al. measured $0.016 \mathrm{~dB} /$ turn for $3-\mu \mathrm{m}$ bend radius ${ }^{[17]}$.

Figure 5 shows TE through and drop responses of a single ring resonator with the following parameters: $\mathrm{WGH}=0.29 \mu \mathrm{m}, \mathrm{WGW}=0.38 \mu \mathrm{m}$, and radius $r=2$ $\mu \mathrm{m}$. It can be seen that the FSR, which is $46 \mathrm{~nm}$ in this case, is significantly larger than those of the filters based on rib waveguides. The extinction ratio (ER) is 12.6 and the $Q$ factor is about 500, much smaller than those reported for rib waveguide based filters ${ }^{[4]}$. For smaller radii, radiation losses become dominant and $\mathrm{ER}$ and $Q$ drops even further, which is demonstrated in Fig. 6 where the TE spectral response of a ring resonator with the ring radius of $1.5 \mu \mathrm{m}$ is shown. The FSR of $\sim 63 \mathrm{~nm}$ is, to the authors' knowledge, the largest reported FSR in SOI platform to date. Although the FSR of the 1.5$\mu \mathrm{m}$ ring is better by approximately $20 \mathrm{~nm}$ compared with the $2.0-\mu \mathrm{m}$ ring, the other figures of merit such as

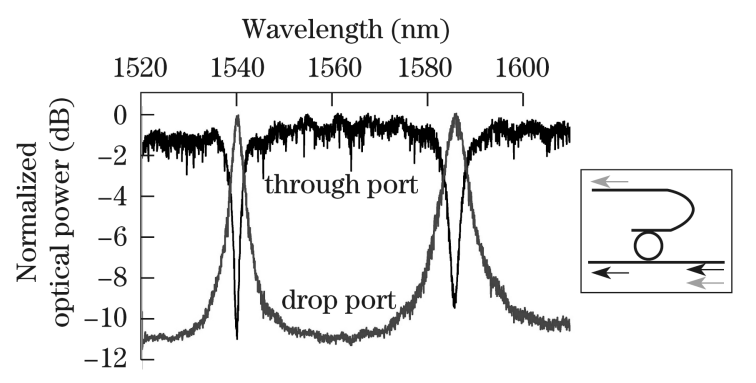

Fig. 5. TE spectral responses at the through and drop ports of a single ring with $\mathrm{WGH}=0.29 \mu \mathrm{m}, \mathrm{WGW}=0.38 \mu \mathrm{m}$, and $r=2 \mu \mathrm{m}$. 


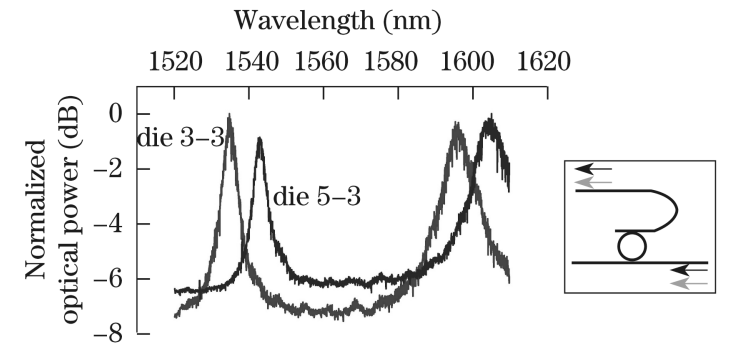

Fig. 6. TE spectral responses at the drop ports of single rings from test chips die3-3 and die5-3. WGH $=0.29 \mu \mathrm{m}$, WGW $=0.38 \mu \mathrm{m}$, and $r=1.5 \mu \mathrm{m}$.

FWHM and $\mathrm{ER}=5.3$ are almost twice better for the $2-\mu \mathrm{m}$ ring.

In addition, there may be an issue with reproducibility of the results considering that some of the dimensional requirements of the devices, such as the separation in the directional coupler and ring radii, reach precision limits of today's fabrication processing tools. This is demonstrated in Fig. 6, where two identical devices exposed to slightly different fabrication processes are compared. This change is meant to give slightly different WGWs and directional coupler separations, but without altering device length (circumference). More precisely, the designed separation in directional couplers is $0.12 \mu \mathrm{m}$ while the predicted separation, on the basis of the fabrication steps applied, is $0.118 \mu \mathrm{m}$ for the first chip, and $0.126 \mu \mathrm{m}$ for the second chip. This highlights one of the issues with the use of small ring resonators for filtering applications. Figure 6 shows the responses of two nominally identical single rings from these two test chips. It can be seen that the responses are separated by $9 \mathrm{~nm}$, which is caused by the separation change of $0.126-0.118=0.008(\mu \mathrm{m})$. This gives a shift of $1 \mathrm{~nm}$ in spectra per $1 \mathrm{~nm}$ change in the WGW and coupler gap, which generally indicates the significant sensitivity of small strip filters.

Thus, a large FSR is a promising feature of SOI strip devices if certain flexibility is allowed for the spectral response stability, and if the polarization insensitivity is not a necessity. An alternative route to improve the FWHM, $\mathrm{ER}$, loss, and reproducibility of the response could be the use of larger resonators coupled in the Vernier configuration (to improve the FSR). We have used this approach and analyzed cascaded strip-racetrack resonators (Fig. 7). Two typical responses are given in Fig. 8. Figure 8(a) is the spectral response of a device that resonates near $1.56 \mu \mathrm{m}(\mathrm{WGW}=0.30 \mu \mathrm{m})$ and Fig. $8(\mathrm{~b})$ is the spectral response of a device that resonates around $1.6 \mu \mathrm{m}$ $(\mathrm{WGW}=0.34 \mu \mathrm{m})$. For comparison, the response of one of the single racetracks has been added to Fig. 8(b) to demonstrate the potential improvement of the FSR by using the Vernier racetracks. It is shown that the Vernier configuration is useful to attenuate the resonances that exist in the response of a single racetrack with a WGW of $0.34 \mu \mathrm{m}$, bend radii of $5 \mu \mathrm{m}$, and length of the coupler of $5 \mu \mathrm{m}$, which were used to build the given Vernier device. However, in order to remove remaining extra peaks and to flatten the resonance at $1.56 \mu \mathrm{m}$, some design and fabrication adjustments need to be performed. Figure 8 indicates that the Vernier configuration could be used in applications where FSR $>60 \mathrm{~nm}$ is required, by

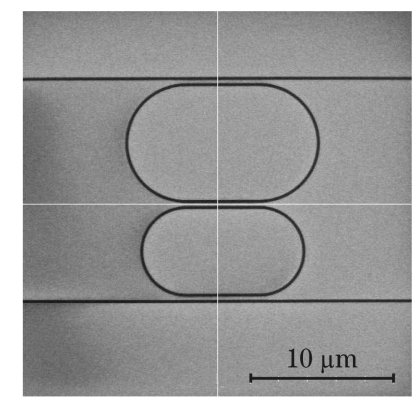

Fig. 7. Scanning electron micrograph of two-level series coupled racetrack resonators with radii of 5 and $4 \mu \mathrm{m}$, length of the directional coupler of $5 \mu \mathrm{m}$, and separation of $0.12 \mu \mathrm{m}$.
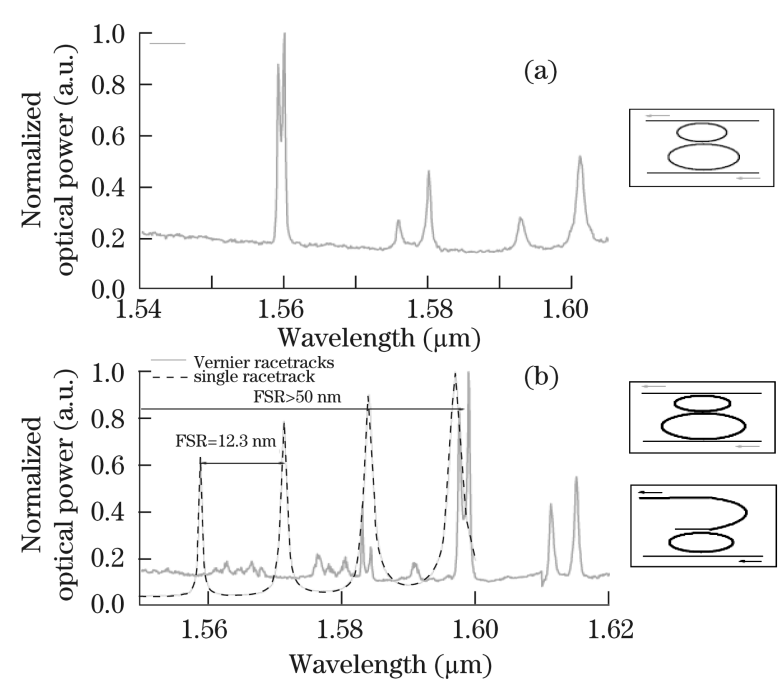

Fig. 8. TM spectral responses at the drop ports of double Vernier racetrack resonator filters with $\mathrm{WGH}=0.29 \mu \mathrm{m}$ and separation of $0.12 \mu \mathrm{m}$. (a) WGW $=0.3 \mu \mathrm{m}, r_{1}=4 \mu \mathrm{m}$, and $r_{2}=3 \mu \mathrm{m}$; (b) WGW $=0.34 \mu \mathrm{m}, r_{1}=5 \mu \mathrm{m}$, and $r_{2}=4 \mu \mathrm{m}$.

employing the racetracks with the individual FSR of only $12 \mathrm{~nm}$.

In conclusion, we have reported single and multiple ring and racetrack resonator filters built upon two main SOI waveguide structures, namely the strip and rib waveguides. The device platforms were discussed from modal and polarization standpoints with the aim to achieve simultaneous operation in single-mode and polarization insensitive regime. The FSR as large as $63 \mathrm{~nm}$ has been achieved by using $1.5-\mu \mathrm{m}$ strip resonators on strip waveguides. However, it is of utmost importance to control the feature sizes of the resonators very tightly, as the coupling efficiency of the directional coupler sections depends strongly on the spacing between the waveguides. By using the same platform, potentially better spectral response can be achieved through cascaded racetrack resonators. Polarization insensitive response has been demonstrated over the FSR of $0.5 \mathrm{~nm}$ by using the rib waveguide platform.

B. Timotijevic is grateful to Intel Corporation for funding and device fabrication. G. Mashanovich would like to acknowledge the support from the Royal Society, UK. This work has been partly supported by the EPSRC (UK Silicon Photonics Programme). 


\section{References}

1. G. T. Reed and A. P. Knights, Silicon Photonics: An Introduction (Wiley, Chichester, 2004).

2. G. T. Reed, (ed.) Silicon Photonics: the State of the Art (Wiley, Chichester, 2008).

3. W. N. Ye, D.-X. Xu, S. Janz, P. Cheben, M.-J. Picard, B. Lamontagne, and N. G. Tarr, J. Lightwave Technol. 23, 1308 (2005).

4. W. R. Headley, G. T. Reed, S. Howe, A. Liu, and M. Paniccia, Appl. Phys. Lett. 85, 5523 (2004).

5. Y. A. Vlasov and S. J. McNab, Opt. Express 12, 1622 (2004).

6. W. Bogaerts, R. Baets, P. Dumon, V. Wiaux, S. Beckx, D. Taillaert, B. Luyssaert, J. Van Campenhout, P. Bienstman, and D. Van Thourhout, J. Lightwave Technol. 23, 401 (2005).

7. K. Petermann, Archiv für Elektronik und übertragungstechnik 30, 139 (1976).

8. W. Bogaerts, D. Taillaert, P. Dumon, D. Van Thourhout, R. Baets, and E. Pluk, Opt. Express 15, 1567 (2007).

9. N. Dagli and C. G. Fonstad, IEEE J. Quantum Electron. 21, 315 (1985).

10. O. Powell, J. Lightwave Technol. 20, 1851 (2002).

11. M. Miloševic, P. S. Matavulj, B. D. Timotijevic, G. T. Reed, and G. Z. Mashanovich, J. Lightwave Technol. 26, 1840 (2008).

12. M. K. Chin and S. T. Ho, J. Lightwave Technol. 16, 1433 (1998).

13. G. Griffel, IEEE Photon. Technol. Lett. 12, 1642 (2000).
14. A. Melloni, F. Morichetti, and M. Martinelli, Opt. Lett. 29, 2785 (2004).

15. F. Y. Gardes, G. T. Reed, N. G. Emerson, and C. E. Png, Opt. Express 13, 8845 (2005).

16. Q. Xu, S. Manipatruni, B. Schmidt, J. Shakya, and M. Lipson, Opt. Express 15, 430 (2007).

17. W. Bogaerts, P. Dumon, D. Van Thourhout, D. Taillaert, P. Jaenen, J. Wouters, S. Beckx, V. Wiaux, and R. G. Baets, IEEE J. Sel. Top. Quantum Eletron. 12, 1394 (2006).

18. K. Yamada, H. Fukuda, T. Watanabe, T. Tsuchizawa, T. Shoji, and S.-I. Itabashi, in Proceedings of IEEE LEOS 2nd Group IV Photonics Conference 186 (2005).

19. T. Tsuchizawa, K. Yamada, H. Fukuda, T. Watanabe, J. Takahashi, M. Takahashi, T. Shoji, E. Tamechika, S.-I. Itabashi, and H. Morita, IEEE J. Sel. Top. Quantum Electron. 11, 232 (2005).

20. P. Dumon, W. Bogaerts, V. Wiaux, J. Wouters, S. Beckx, J. Van Campenhout, D. Taillaert, B. Luyssaert, P. Bienstman, D. Van Thourhout, and R. Baets, IEEE Photon. Technol. Lett. 16, 1328 (2004).

21. B. D. Timotijevic, "Auto-regressive optical filters in silicon-on-insulator waveguides" $\mathrm{PhD}$ thesis (University of Surrey, 2007).

22. T. Tsuchizawa, T. Watanabe, E. Tamechika, T. Shoji, K. Yamada, J. Takahashi, S. Uchiyama, S. Itabashi, and H. Morita, in Proceedings of the 15th Annual IEEE LEOS Meeting 287 (2002).

23. K. K. Lee, D. R. Lim, L. C. Kimerling, J. Shin, and F. Cerrina, Opt. Lett. 26, 1888 (2001). 